\title{
Pre-hospital critical care anaesthesiologists and traumatic brain injury-guideline adherence
}

\author{
Leif Rognås ${ }^{1,2,3^{*}}$, Troels Martin Hansen ${ }^{2}$, Hans Kirkegaard ${ }^{3}$, Else Tønnesen ${ }^{4}$ \\ From London Trauma Conference 2013 \\ London, UK. 10-13 December 2013
}

\section{Background}

Guidelines recommend that brain trauma patients with a Glasgow Coma Scale score $<9$ should have an airway established. Also, $\mathrm{SpO}_{2}>90 \%$, systolic blood pressure $>90 \mathrm{mmHg}$ and end-tidal $\mathrm{CO}_{2}$ between 4.5 and $5.3 \mathrm{kPa}$ is advised [1]. The objectives were to investigate guideline adherence, reasons for non-adherence and the incidences of complications related to pre-hospital advanced airway management in traumatic brain injury patients.

\section{Materials and methods}

We prospectively collected data [2] from eight anaesthesiologist-staffed pre-hospital critical care teams in the Central Denmark Region according to the Utstein-style template [3].

\section{Results}

Among 1081 consecutive pre-hospital advanced airway management patients, we identified 54 with a traumatic brain injury and an initial Glasgow Coma Scale score $<9$. Guideline adherence regarding airway management was $92.6 \%$. Reasons for non-adherence were patient's condition, anticipated difficult airway management and short distance to the emergency department. Following rapid sequence intubation, $11.4 \%$ suffered an oxygen saturation $<90 \%, 9.1 \%$ had a first post-rapid sequence intubation systolic blood pressure $<90 \mathrm{mmHg}$ and $48.9 \%$ had a first post-rapid sequence intubation systolic blood pressure $<120 \mathrm{mmHg}$. The incidence of hypertension following pre-hospital rapid sequence intubation was $4.5 \%$. The incidence of post-endotracheal intubation hyperventilation was $71.1 \%$.

\section{Conclusion}

The adherence to airway management guidelines was high. The incidences of post-rapid sequence intubation hypoxia and systolic blood pressure $<90$ compare to results reported from other physician-staffed pre-hospital services. The incidence of systolic blood pressure $<120$ as well as that of hyperventilation following pre-hospital endotracheal intubation in traumatic brain injury patients call for a change in our current practice.

\section{Authors' details}

'Department of Research and Development, Norwegian Air Ambulance Foundation, Drøbak, Norway. ${ }^{2}$ Pre-hospital Critical Care Team, Aarhus University Hospital, Aarhus, Denmark. ${ }^{3}$ Centre for Emergency Medicine Research, Aarhus University Hospital, Aarhus, Denmark. ${ }^{4}$ Department of Anaesthesiology, Aarhus University Hospital Aarhus, Denmark.

Published: 7 July 2014

\section{References}

1. Badjatia N, Carney N, Crocco TJ, et al: Guidelines for prehospital management of traumatic brain injury. Prehospital emergency care : official journal of the National Association of EMS Physicians and the National Association of State EMS Directors , 2 2008, 12(Suppl 1):S1-52.

2. Rognas L, Hansen TM, Kirkegaard H, et al: Pre-hospital advanced airway management by experienced anaesthesiologists: a prospective descriptive study. Scandinavian journal of trauma, resuscitation and emergency medicine 2013, 21(1):58.

3. Sollid SJ, Lockey D, Lossius HM: A consensus-based template for uniform reporting of data from pre-hospital advanced airway management. Scandinavian journal of trauma, resuscitation and emergency medicine 2009, 17:58.

doi:10.1186/1757-7241-22-S1-P7

Cite this article as: Rognås et al:: Pre-hospital critical care anaesthesiologists and traumatic brain injury-guideline adherence. Scandinavian Journal of Trauma, Resuscitation and Emergency Medicine 2014 22(Suppl 1):P7. 